\title{
Commonly used medications cause clinically relevant decreases in serum PSA levels
}

The use of PSA measurement as a screening tool for prostate cancer is a widespread practice. However, many factors can influence PSA levels, including age, the presence of prostatitis, and prostate size. In addition, studies have suggested that several medications might affect PSA, such as NSAIDs and statins. Chang and colleagues at the Stanford University School of Medicine analyzed data from the National Health and Nutrition Examination Survey to study the effects of 10 classes of commonly used medications on PSA levels in 1,864 men aged $\geq 40$ years.

The medication classes studied were metabolic agents (statins and sulfonylureas), cardiovascular agents ( $\beta$-blockers, calcium channel blockers, angiotensin-converting-enzyme [ACE] inhibitors and thiazide diuretics), proton pump inhibitors, NSAIDs, selective serotonin reuptake inhibitors, and a-blockers. In addition, combinations of medications frequently administered together in fixed-dose formulations or as concurrent prescriptions were investigated. The effects of these medications were adjusted for a range of clinical and sociodemographic factors that might have affected PSA levels.

\section{4 ...the underlying} mechanisms driving these

\section{associations are unclear... 77}

Use of NSAIDS, statins or thiazide diuretics was inversely correlated with PSA level $(P<0.05)$ : 5 years' regular use was associated with $6 \%, 13 \%$ and $26 \%$ lower PSA levels, respectively. In addition, fixed-dose combination of thiazide diuretics with $\beta$-blockers or ACE inhibitors, and concurrently prescribed statins with $\beta$-blockers, ACE inhibitors or thiazide diuretics, were all associated with significantly decreased PSA levels
$(P<0.05)$. The effects of statins on PSA seemed to be negated by concurrent use of calcium channel blockers.

"In the current study, we found that men taking NSAIDs, statins or thiazide diuretics had statistically and clinically significant decreases in serum levels of PSA compared to men not on those medications," says Steven Chang, lead author of the study, "however, the underlying mechanisms driving these associations are unclear at this time." Further studies will be required to determine whether these medicationassociated PSA decreases affect the development of prostate cancer or the interpretation of PSA screening results.

Nick Warde

Original article Chang, S. L. et al. Impact of common medications on serum total prostate-specific antigen levels: analysis of the National Health and Nutrition Examination Survey. J. Clin. Oncol. 28, 3951-3957 (2010) 